Naxi mal si ngul ar i nt egral s on product homogeneous gr oups

\begin{tabular}{|l|l|}
\hline 著者 & Di ng Yong, Sat o Shui chi \\
\hline $\begin{array}{l}\text { j our nal or } \\
\text { publ i cat i on t i t l e }\end{array}$ & St udi a Nat hemat i ca \\
\hline vol une & 222 \\
\hline number & 1 \\
\hline page r ange & $41-49$ \\
\hline year & 2014 01- 01 \\
\hline URL & ht t p: //hdl . handl e. net /2297/43048 \\
\hline
\end{tabular}




\title{
MAXIMAL SINGULAR INTEGRALS ON PRODUCT HOMOGENEOUS GROUPS
}

\author{
YONG DING AND SHUICHI SATO
}

\begin{abstract}
We prove $L^{p}$ boundedness for $p \in(1, \infty)$ of maximal singular integral operators with rough kernels on product homogeneous groups under a sharp integrability condition of the kernels.
\end{abstract}

\section{INTRODUCTION}

Let $\mathbb{R}^{d}, d \geq 2$, be the $d$-dimensional Euclidean space. We assume that $\mathbb{R}^{d}$ is also equipped with a homogeneous group structure, where multiplication is given by a polynomial mapping; the underlying manifold is $\mathbb{R}^{d}$ itself. We also write $\mathbb{R}^{d}=\mathbb{H}$. Thus, $\mathbb{H}$ is associated with a dilation group $\left\{A_{t}\right\}_{t>0}$ of automorphisms of the group structure such that

$$
A_{t} x=\left(t^{a_{1}} x_{1}, t^{a_{2}} x_{2}, \ldots, t^{a_{d}} x_{d}\right), \quad x=\left(x_{1}, \ldots, x_{d}\right) \in \mathbb{H},
$$

where real numbers $a_{1}, \ldots, a_{d}$ satisfy $0<a_{1} \leq a_{2} \leq \cdots \leq a_{d}$ (see [10], [21], [18], [6] and [11, Section 2 of Chapter 1]). So, we have, for each $t>0$,

$$
A_{t}(x y)=\left(A_{t} x\right)\left(A_{t} y\right), \quad x, y \in \mathbb{H} \text {. }
$$

Consequently, $\mathbb{H}$ is endowed with both the Euclidean structure and a homogeneous nilpotent Lie group structure. The group law of $\mathbb{H}$ is given by a polynomial mapping which conforms to the Campbell-Hausdorff formula in a corresponding Lie algebra via an exponential map and the action of the automorphism family $\left\{A_{t}\right\}$. We note that the identity is the origin 0 and $x^{-1}=-x$; furthermore, Lebesgue measure is bi-invariant Haar measure.

Let us recall a norm function $r(x)$ associated with $\left\{A_{t}\right\}$. The function $r(x)$, which is non-negative and vanishes only at the origin, satisfies that $r\left(A_{t} x\right)=\operatorname{tr}(x)$ for $t>0$ and $x \in \mathbb{R}^{d}$. We assume that $r(x)$ is even, continuous on $\mathbb{R}^{d}$ and smooth in $\mathbb{R}^{d} \backslash\{0\}$, and also that the unit sphere $\Sigma_{d}=\left\{x \in \mathbb{R}^{d}: r(x)=1\right\}$ defined by the norm function coincides with the unit sphere $S^{d-1}=\left\{x \in \mathbb{R}^{d}:|x|=1\right\}$, where $|x|$ denotes the Euclidean norm. Let $\gamma=a_{1}+\cdots+a_{d}$ (the homogeneous dimension of $\left.\mathbb{H}\right)$. We shall

2010 Mathematics Subject Classification. Primary 42B20.

Key words and phrases. Multiple singular integrals, homogeneous groups, maximal singular integrals. 
use the formula:

$$
\int_{\mathbb{R}^{d}} f(x) d x=\int_{0}^{\infty} \int_{\Sigma_{d}} f\left(A_{t} \theta\right) t^{\gamma-1} d S_{d}(\theta) d t, \quad d S_{d}=\omega d \sigma_{d},
$$

where $\omega$ is a strictly positive $C^{\infty}$ function on $\Sigma_{d}$ and $d \sigma_{d}$ is the Lebesgue surface measure on $\Sigma_{d}$. See $[18,6]$ and also $[3,10,11,14,19,20,21]$ for more details and related results.

We consider a function $\Omega$ which is locally integrable in $\mathbb{R}^{d} \backslash\{0\}$ and homogeneous of degree 0 with respect to the dilation group $\left\{A_{t}\right\}$, that is, $\Omega\left(A_{t} x\right)=\Omega(x)$ for $x \neq 0, t>0$. We assume the cancellation property:

$$
\int_{\Sigma_{d}} \Omega(\theta) d S_{d}(\theta)=0
$$

Convolution on $\mathbb{H}$ is defined by

$$
f * g(x)=\int_{\mathbb{H}} f(y) g\left(y^{-1} x\right) d y .
$$

Let

$$
T f(x)=\text { p.v. } f * K(x)=\text { p.v. } \int_{\mathbb{H}} f(y) K\left(y^{-1} x\right) d y
$$

for appropriate functions $f$, where $K(x)=\Omega\left(x^{\prime}\right) r(x)^{-\gamma}, x^{\prime}=A_{r(x)^{-1}} x$ for $x \neq 0$. We also define the maximal singular integral operator

$$
T_{*} f(x)=\sup _{\epsilon>0}\left|\int_{r(y)>\epsilon} f\left(x y^{-1}\right) K(y) d y\right| .
$$

Then the following results are known.

Theorem A ([21]). If $\Omega \in L \log L\left(\Sigma_{d}\right)$ with (1.1) and $T f$ is as in (1.2), then $T$ is bounded on $L^{p}(\mathbb{H})$ for all $p \in(1, \infty)$.

Theorem B ([18]). Let $T_{*} f$ be defined as in (1.3) with $\Omega \in L \log L\left(\Sigma_{d}\right)$ satisfying (1.1). Let $p \in(1, \infty)$. Then the operator $T_{*}$ is bounded on $L^{p}(\mathbb{H})$.

We refer to $[4,12,13,14,15,16]$ for relevant results.

Part of a theory of Duoandikoetxea and Rubio de Francia [8] for singular integrals on the Euclidean spaces has been generalized to the case of homogeneous groups by [18]. The arguments of [18] replace Fourier transform estimates by a variant of Tao's $L^{2}$ estimates via convolution (see [21]). As a result, [18] proved Theorem B and some weighted estimates, and also gave another proof of Theorem A.

Also, it has been shown in [6] that the theory of [18] extends to the case of product homogeneous groups to treat multiple singular integrals. Let $\mathbb{R}^{n}=\mathbb{R}^{n_{1}} \times \mathbb{R}^{n_{2}}$ be a product homogeneous group with $\mathbb{R}^{n_{1}}=\mathbb{H}_{1}$, $\mathbb{R}^{n_{2}}=\mathbb{H}_{2}$, where $n=n_{1}+n_{2}$ and $\mathbb{H}_{1}, \mathbb{H}_{2}$ are homogeneous groups with 
dilations $A_{t}^{(1)}, A_{t}^{(2)}$ and norm functions $r_{1}, r_{2}$, respectively. We consider a function $\Omega$ in $L^{1}\left(\Sigma_{n_{1}} \times \Sigma_{n_{2}}\right)$ which satisfies

$$
\begin{aligned}
& \int_{\Sigma_{n_{1}}} \Omega(u, v) d S_{n_{1}}(u)=0, \quad \text { for all } v \in \Sigma_{n_{2}}, \\
& \int_{\Sigma_{n_{2}}} \Omega(u, v) d S_{n_{2}}(v)=0, \quad \text { for all } u \in \Sigma_{n_{1}} .
\end{aligned}
$$

Define

$$
K(u, v)=r_{1}(u)^{-\gamma_{1}} r_{2}(v)^{-\gamma_{2}} \Omega\left(u^{\prime}, v^{\prime}\right), \quad u^{\prime}=A_{r_{1}(u)^{-1}}^{(1)} u, v^{\prime}=A_{r_{2}(v)^{-1}}^{(2)} v,
$$

where $\gamma_{1}$ and $\gamma_{2}$ are the homogeneous dimensions of $\mathbb{H}_{1}$ and $\mathbb{H}_{2}$, respectively. We consider the multiple singular integral

$$
T f(x, y)=\text { p.v. } f * K(x, y)=\text { p.v. } \int_{\mathbb{H}_{1} \times \mathbb{H}_{2}} f\left(x u^{-1}, y v^{-1}\right) K(u, v) d u d v .
$$

Then the following result is proved in [6].

Theorem C. Let $T$ be defined as in (1.6) with $\Omega$ in $L(\log L)^{2}\left(\Sigma_{n_{1}} \times \Sigma_{n_{2}}\right)$ satisfying (1.4) and (1.5). Let $1<p<\infty$. The operator $T$ is then bounded on $L^{p}\left(\mathbb{H}_{1} \times \mathbb{H}_{2}\right)$.

We can find in [2] the optimality of the $L(\log L)^{2}$ integrability condition for multiple singular integrals with Euclidean convolution.

Let us recall that the maximal singular integral is defined by

$$
T_{*} f(x, y)=\sup _{\substack{\epsilon_{1}>0, \epsilon_{2}>0}}\left|\int_{\substack{r_{1}(u)>\epsilon_{1}, r_{2}(v)>\epsilon_{2}}} f\left(x u^{-1}, y v^{-1}\right) K(u, v) d u d v\right| .
$$

In this note we shall prove the following.

Theorem 1. Let $T_{*}$ be defined as in (1.7). Suppose that $\Omega$ is in $L(\log L)^{2}\left(\Sigma_{n_{1}} \times\right.$ $\left.\Sigma_{n_{2}}\right)$ and satisfies (1.4), (1.5). Then $T_{*}$ is bounded on $L^{p}\left(\mathbb{H}_{1} \times \mathbb{H}_{2}\right)$ for all $p \in(1, \infty)$.

Previous works concerning singular integrals on product of Euclidean spaces can be found in $[1,2,7,9]$. Theorem 1 is an analogue of a result of [2] for multiple singular integrals on product homogeneous groups.

Similarly to the proof of Theorem $C$ in [6], we use extrapolation arguments in proving Theorem 1 by applying the following result.

Theorem 2. Let $1<s \leq 2$. Suppose that $\Omega$ is in $L^{s}\left(\Sigma_{n_{1}} \times \Sigma_{n_{2}}\right)$ and satisfies (1.4), (1.5). Then, for $1<p<\infty$ we have

$$
\left\|T_{*} f\right\|_{p} \leq C_{p}(s-1)^{-2}\|\Omega\|_{s}\|f\|_{p}
$$


with a constant $C_{p}$ independent of $s$ and $\Omega$.

Let $\Omega$ be as in Theorem 1 . Then there exist a sequence $\left\{\Omega_{k}\right\}$ of functions in $L^{1}\left(\Sigma_{n_{1}} \times \Sigma_{n_{2}}\right)$ and a sequence $\left\{c_{k}\right\}$ of non-negative real numbers such that each $\Omega_{k}$ satisfies (1.4) and (1.5), $\sup _{k \geq 1}\left\|\Omega_{k}\right\|_{1+1 / k} \leq 1, \sum_{k=1}^{\infty} k^{2} c_{k}<\infty$, and

$$
\Omega=\sum_{k=1}^{\infty} c_{k} \Omega_{k}
$$

Theorem 1 easily follows from this decomposition of $\Omega$ and Theorem 2 (see $[17,15,16])$.

In Section 2 we recall some preliminary results from [6]. We shall prove Theorem 2 in Section 3 by using results of $[6,5,18]$; similar arguments, via Fourier transform estimates, for singular integrals with Euclidean convolution can be found in $[1,2]$.

\section{Preliminaries}

Let $\rho \geq 2$ and let $\psi_{j} \in C_{0}^{\infty}(\mathbb{R}), j \in \mathbb{Z}$ (the set of integers), be such that

$$
\begin{gathered}
\operatorname{supp}\left(\psi_{j}\right) \subset\left\{t \in \mathbb{R}: \rho^{j} \leq t \leq \rho^{j+2}\right\}, \quad \psi_{j} \geq 0 \\
(\log 2) \sum_{j \in \mathbb{Z}} \psi_{j}(t)=1 \quad \text { for } t>0
\end{gathered}
$$

furthermore,

$$
\left|(d / d t)^{m} \psi_{j}(t)\right| \leq c_{m}|t|^{-m} \text { for } m=0,1,2, \ldots,
$$

where $c_{m}$ is a constant independent of $\rho$; we note that this is possible since $\rho \geq 2$.

Suppose that $F$ belongs to $L^{1}\left(\mathbb{H}_{1} \times \mathbb{H}_{2}\right)$ with support in $D_{0}$, where $D_{0}=$ $D_{0}^{(1)} \times D_{0}^{(2)}$,

$$
D_{0}^{(1)}=\left\{x \in \mathbb{H}_{1}: 1 \leq r_{1}(x) \leq 2\right\}, \quad D_{0}^{(2)}=\left\{y \in \mathbb{H}_{2}: 1 \leq r_{2}(y) \leq 2\right\} .
$$

Let $\delta_{s}^{(1)} f(x)=s^{-\gamma_{1}} f\left(\left(A_{s}^{(1)}\right)^{-1} x\right), \delta_{t}^{(2)} g(y)=t^{-\gamma_{2}} g\left(\left(A_{t}^{(2)}\right)^{-1} y\right)$. Define $\delta_{s, t}=$ $\delta_{s}^{(1)} \otimes \delta_{t}^{(2)}$ and let

$$
S_{j, k} F(x, y)=\int_{0}^{\infty} \int_{0}^{\infty} \psi_{j}(s) \psi_{k}(t) \delta_{s, t} F(x, y) \frac{d s}{s} \frac{d t}{t} .
$$

Then $\sum_{j, k \in \mathbb{Z}} S_{j, k} K_{0}=K$, where

$$
K_{0}(x, y)=\left\{\begin{array}{rr}
K(x, y), & (x, y) \in D_{0}, \\
0, & \text { otherwise. }
\end{array}\right.
$$

For $s \geq 1$, let $L^{s}\left(D_{0}\right)$ denote the subspace of $L^{s}\left(\mathbb{H}_{1} \times \mathbb{H}_{2}\right)$ consisting of functions $F$ with support in $D_{0}$. Define

$$
M_{F} f(x, y)=\sup _{j, k \in \mathbb{Z}}\left|f * S_{j, k}(|F|)(x, y)\right|
$$


for $F \in L^{s}\left(D_{0}\right)$. The following result is Lemma 8 of [6].

Lemma 1. Let $p>1$. Suppose that $s \in(1,2], \rho=2^{s^{\prime}}, s^{\prime}=s /(s-1)$, and $F \in L^{s}\left(D_{0}\right)$. Then,

$$
\left\|M_{F} f\right\|_{p} \leq C_{p}(s-1)^{-2}\|F\|_{s}\|f\|_{p}
$$

with a positive constant $C_{p}$ independent of $s$ and $F$.

Also, we need another result of [6].

Lemma 2. Let $1<s \leq 2$. Suppose that $\Omega$ belongs to $L^{s}\left(\Sigma_{n_{1}} \times \Sigma_{n_{2}}\right)$ and satisfies (1.4) and (1.5). Let

$$
R f(x, y)=\sup _{\ell, m \in \mathbb{Z}}\left|\sum_{j=\ell}^{\infty} \sum_{k=m}^{\infty} f * S_{j, k} K_{0}(x, y)\right|,
$$

where $K_{0}$ is defined by (2.2) and $S_{j, k} K_{0}$ is as in (2.1) with $K_{0}$ in place of $F$. Let $\rho=2^{s^{\prime}}$. Then, for $p \in(1, \infty)$ there exists a positive constant $C_{p}$ independent of $s \in(1,2]$ and $\Omega \in L^{s}$ such that

$$
\|R f\|_{p} \leq C_{p} A(s, \Omega)\|f\|_{p}
$$

where $A(s, \Omega)=(s-1)^{-2}\|\Omega\|_{s}$.

This is Proposition 2 of [6].

\section{Proof of Theorem 2.}

We first note that

$S_{j, k} K_{0}(x, y)=r_{1}(x)^{-\gamma_{1}} r_{2}(y)^{-\gamma_{2}} \Omega\left(x^{\prime}, y^{\prime}\right) \int_{1 / 2}^{1} \psi_{j}\left(s r_{1}(x)\right) \frac{d s}{s} \int_{1 / 2}^{1} \psi_{k}\left(\operatorname{tr}_{2}(y)\right) \frac{d t}{t}$,

where $x^{\prime}=A_{r_{1}(x)^{-1}}^{(1)} x, y^{\prime}=A_{r_{2}(y)^{-1}}^{(2)} y$. From this we easily see that

$$
\operatorname{supp}\left(S_{j, k} K_{0}\right) \subset\left\{\rho^{j} \leq r_{1}(x) \leq 2 \rho^{j+2}\right\} \times\left\{\rho^{k} \leq r_{2}(y) \leq 2 \rho^{k+2}\right\} .
$$

Thus, if $\ell, m \in \mathbb{Z}$ are determined by the conditions $\rho^{\ell+2} \leq \epsilon<\rho^{\ell+3}, \rho^{m+2} \leq$ $\delta<\rho^{m+3}$ and if $f$ is a compactly supported smooth function, we have

$$
\begin{aligned}
& \int_{\substack{r_{1}(u)>\epsilon, r_{2}(v)>\delta}} f\left(x u^{-1}, y v^{-1}\right) K(u, v) d u d v \\
& =\sum_{\substack{j \geq \ell \\
k \geq m}} \int_{\substack{r_{1}(u)>\epsilon, r_{2}(v)>\delta}} f\left(x u^{-1}, y v^{-1}\right) S_{j, k} K_{0}(u, v) d u d v \\
& =A_{\epsilon, \delta} f(x, y)+B_{\epsilon, \delta} f(x, y)+C_{\epsilon, \delta} f(x, y)+D_{\epsilon, \delta} f(x, y),
\end{aligned}
$$


where

$$
\begin{aligned}
A_{\epsilon, \delta} f(x, y) & =\sum_{\substack{j>\ell+3, k>m+3}} \int_{\mathbb{H}_{1} \times \mathbb{H}_{2}} f\left(x u^{-1}, y v^{-1}\right) S_{j, k} K_{0}(u, v) d u d v \\
& =\sum_{\substack{j>\ell+3, k>m+3}} f * S_{j, k} K_{0}(x, y) \\
B_{\epsilon, \delta} f(x, y) & =\sum_{\substack{\ell+3 \geq j \geq \ell \\
k>m+3}} \int_{\left\{r_{1}(u)>\epsilon\right\} \times \mathbb{H}_{2}} f\left(x u^{-1}, y v^{-1}\right) S_{j, k} K_{0}(u, v) d u d v \\
C_{\epsilon, \delta} f(x, y) & =\sum_{\substack{j>\ell+3, m+3 \geq k \geq m}} \int_{\mathbb{H}_{1} \times\left\{r_{2}(v)>\delta\right\}} f\left(x u^{-1}, y v^{-1}\right) S_{j, k} K_{0}(u, v) d u d v \\
D_{\epsilon, \delta} f(x, y) & =\sum_{\substack{\ell+3>j>\ell, m+3 \geq k \geq m}} \int_{\substack{r_{1}(u)>\epsilon, r_{2}(v)>\delta}} f\left(x u^{-1}, y v^{-1}\right) S_{j, k} K_{0}(u, v) d u d v .
\end{aligned}
$$

Let

$$
\begin{array}{ll}
A_{*} f(x, y)=\sup _{\epsilon, \delta>0}\left|A_{\epsilon, \delta} f(x, y)\right|, & B_{*} f(x, y)=\sup _{\epsilon, \delta>0}\left|B_{\epsilon, \delta} f(x, y)\right|, \\
C_{*} f(x, y)=\sup _{\epsilon, \delta>0}\left|C_{\epsilon, \delta} f(x, y)\right|, & D_{*} f(x, y)=\sup _{\epsilon, \delta>0}\left|D_{\epsilon, \delta} f(x, y)\right| .
\end{array}
$$

Then, (3.1) implies

$$
T_{*} f(x, y) \leq A_{*} f(x, y)+B_{*} f(x, y)+C_{*} f(x, y)+D_{*} f(x, y) .
$$

Let $\rho=2^{s^{\prime}}$. Since $A_{*} f \leq R f$, by Lemma 2 we have

$$
\left\|A_{*} f\right\|_{p} \leq C_{p} A(s, \Omega)\|f\|_{p} .
$$

Also, since $D_{*} f \leq C M_{K_{0}}(|f|)$, Lemma 1 implies

$$
\left\|D_{*} f\right\|_{p} \leq C\left\|M_{K_{0}}(|f|)\right\|_{p} \leq C_{p} A(s, \Omega)\|f\|_{p} .
$$

To estimate $B_{*} f$, we note that

$$
\begin{aligned}
& \left|B_{\epsilon, \delta} f(x, y)\right| \\
& \quad \leq \sum_{\ell+3 \geq j \geq \ell} \int_{\rho^{\ell} \leq r_{1}(u) \leq 2 \rho^{\ell+5}}\left|\sum_{k>m+3} \int_{\mathbb{H}_{2}} f\left(x u^{-1}, y v^{-1}\right) S_{j, k} K_{0}(u, v) d v\right| d u .
\end{aligned}
$$

By changing variables with respect to $u$, we see that the right hand side is equal to

$$
\sum_{\ell+3 \geq j \geq \ell} \int_{\rho^{\ell}}^{2 \rho^{\ell+5}} \int_{\Sigma_{n_{1}}}\left|\sum_{k>m+3} F_{k}(x, y, s, \theta)\right| \Psi_{j}(s) \frac{d s}{s} d S_{n_{1}}(\theta),
$$

where

$$
F_{k}(x, y, s, \theta)=\int_{\mathbb{H}_{2}} f\left(x\left(A_{s}^{(1)} \theta\right)^{-1}, y v^{-1}\right) \Omega\left(\theta, v^{\prime}\right) r_{2}(v)^{-\gamma_{2}} \Psi_{k}\left(r_{2}(v)\right) d v,
$$


$\Psi_{k}(t)=\int_{1 / 2}^{1} \psi_{k}(r t) d r / r$. Thus, since $0 \leq \Psi_{j}(s) \leq 1$, we have

$$
\left|B_{\epsilon, \delta} f(x, y)\right| \leq C \int_{\rho^{\ell}}^{2 \rho^{\ell+5}} \int_{\Sigma_{n_{1}}}\left|\sum_{k>m+3} F_{k}(x, y, s, \theta)\right| \frac{d s}{s} d S_{n_{1}}(\theta) .
$$

We write

$$
K_{\theta}^{(2)}(v)=K_{0}(\theta, v), \quad S_{k}^{(2)} K_{\theta}^{(2)}(v)=\int_{0}^{\infty} \psi_{k}(t) \delta_{t}^{(2)} K_{\theta}^{(2)}(v) \frac{d t}{t} .
$$

Then

$$
F_{k}(x, y, s, \theta)=f\left(x\left(A_{s}^{(1)} \theta\right)^{-1}, \cdot\right) *_{(2)} S_{k}^{(2)} K_{\theta}^{(2)}(y),
$$

where $*_{(2)}$ denotes the convolution on $\mathbb{H}_{2}$. Consequently,

$$
\begin{aligned}
& \left|B_{\epsilon, \delta} f(x, y)\right| \\
& \leq C \int_{\rho^{\ell}}^{2 \rho^{\ell+5}} \int_{\Sigma_{n_{1}}}\left|\sum_{k>m+3} f\left(x\left(A_{s}^{(1)} \theta\right)^{-1}, \cdot\right) *(2) S_{k}^{(2)} K_{\theta}^{(2)}(y)\right| \frac{d s}{s} d S_{n_{1}}(\theta) .
\end{aligned}
$$

Let

$$
R_{\theta}^{(2)} g(y)=\sup _{m \in \mathbb{Z}}\left|\sum_{k>m} g *_{(2)} S_{k}^{(2)} K_{\theta}^{(2)}(y)\right|
$$

for $g$ on $\mathbb{H}_{2}$. We write $f_{x}(y)=f(x, y)$ when considering $f(x, y)$ as a function on $\mathbb{H}_{2}$ fixing $x$; similarly, we write $f_{y}(x)=f(x, y)$. Define

$$
F^{\theta}(x, y)=R_{\theta}^{(2)} f_{x}(y)
$$

Then, using (3.5), we have

$$
\begin{aligned}
\left|B_{*} f(x, y)\right| & \leq C \sup _{\ell \in \mathbb{Z}} \int_{\rho^{\ell}}^{2 \rho^{\ell+5}} \int_{\Sigma_{n_{1}}} F^{\theta}\left(x\left(A_{s}^{(1)} \theta\right)^{-1}, y\right) \frac{d s}{s} d S_{n_{1}}(\theta) \\
& \leq C \log \rho \int_{\Sigma_{n_{1}}} M_{\theta}^{(1)} F_{y}^{\theta}(x) d S_{n_{1}}(\theta),
\end{aligned}
$$

where

$$
M_{\theta}^{(1)} h(x)=\sup _{t>0} \frac{1}{t} \int_{0}^{t}\left|h\left(x\left(A_{s}^{(1)} \theta\right)^{-1}\right)\right| d s
$$

for $h$ on $\mathbb{H}_{1}$. The last inequality of (3.6) can be seen as follows. Take a positive integer $d$ such that $2^{d} \leq 2 \rho^{5}<2^{d+1}$. Then

$$
\begin{aligned}
\int_{\rho^{\ell}}^{2 \rho^{\ell+5}}\left|h\left(x\left(A_{s}^{(1)} \theta\right)^{-1}\right)\right| \frac{d s}{s} & \leq \sum_{i=0}^{d} \int_{2^{i} \rho^{\ell}}^{2^{i+1} \rho^{\ell}}\left|h\left(x\left(A_{s}^{(1)} \theta\right)^{-1}\right)\right| \frac{d s}{s} \\
& \leq \sum_{i=0}^{d} 2 M_{\theta}^{(1)} h(x)=2(d+1) M_{\theta}^{(1)} h(x) \\
& \leq C(\log \rho) M_{\theta}^{(1)} h(x),
\end{aligned}
$$

since $d \sim \log \rho$. This implies what we need. 
By M. Christ [5] $M_{\theta}^{(1)}$ is bounded on $L^{p}, p>1$, with a bound independent of $\theta$. Thus, using (3.6) and the Minkowski inequality we have

$$
\left\|B_{*} f\right\|_{p} \leq C(\log \rho) \int_{\Sigma_{n_{1}}}\left\|F^{\theta}\right\|_{p} d S_{n_{1}}(\theta) .
$$

By Lemma 9 of [18] with $\rho=2^{s^{\prime}}$, we have

$$
\left\|R_{\theta}^{(2)} g\right\|_{p} \leq C_{p}(\log \rho)\left(\int_{\Sigma_{n_{2}}}|\Omega(\theta, \omega)|^{s} d S_{n_{2}}(\omega)\right)^{1 / s}\|g\|_{p}
$$

Thus

$$
\left\|F_{x}^{\theta}\right\|_{p} \leq C_{p}(\log \rho)\left(\int_{\Sigma_{n_{2}}}|\Omega(\theta, \omega)|^{s} d S_{n_{2}}(\omega)\right)^{1 / s}\left\|f_{x}\right\|_{p} .
$$

Using this in (3.7) and noting $\left\|F^{\theta}\right\|_{p}=\left(\int\left\|F_{x}^{\theta}\right\|_{p}^{p} d x\right)^{1 / p}$, we have

$$
\begin{aligned}
\left\|B_{*} f\right\|_{p} & \leq C_{p}(\log \rho)^{2} \int_{\Sigma_{n_{1}}}\left(\int_{\Sigma_{n_{2}}}|\Omega(\theta, \omega)|^{s} d S_{n_{2}}(\omega)\right)^{1 / s} d S_{n_{1}}(\theta)\|f\|_{p} \\
& \leq C_{p}(\log \rho)^{2}\|\Omega\|_{s}\|f\|_{p}
\end{aligned}
$$

where the last inequality follows from Hölder's inequality.

Similarly, we have

$$
\left\|C_{*} f\right\|_{p} \leq C_{p}(\log \rho)^{2}\|\Omega\|_{s}\|f\|_{p} .
$$

Combining (3.2), (3.3), (3.4), (3.8) and (3.9), we get the conclusion of Theorem 2.

\section{REFERENCES}

[1] H. Al-Qassem and Y. Pan, $L^{p}$ boundedness for singular integrals with rough kernels on product domains, Hokkaido Math. J. 31 (2002), 555-613.

[2] A. Al-Salman, H. Al-Qassem and Y. Pan, Singular integrals on product domains, Indiana Univ. Math. J., 55 (2006), 369-387.

[3] A. P. Calderón and A. Torchinsky, Parabolic maximal functions associated with a distribution, Advances in Math. 16 (1975), 1-64.

[4] A. P. Calderón and A. Zygmund, On singular integrals, Amer. J. Math. 78 (1956), 289-309.

[5] M. Christ, Hilbert transforms along curves I. Nilpotent groups, Ann. of Math. 122 (1985), 575-596.

[6] Y. Ding and S. Sato, Singular integrals on product homogeneous groups, Integr. Equ. Oper. Theory, 76 (2013), 55-79.

[7] J. Duoandikoetxea, Multiple singular integrals and maximal functions along hypersurfaces, Ann. Inst. Fourier 36 (1986), 185-206.

[8] J. Duoandikoetxea and J. L. Rubio de Francia, Maximal and singular integral operators via Fourier transform estimates, Invent. Math. 84 (1986), 541-561.

[9] R. Fefferman and E. M. Stein, Singular integrals on product spaces, Adv. in Math. 45 (1982), 117-143.

[10] G. B. Folland and E. M. Stein, Hardy spaces on homogeneous groups, Princeton Univ. Press, Princeton, N.J. 1982. 
[11] A. Nagel and E. M. Stein, Lectures on pseudo-differential operators, Mathematical Notes 24, Princeton University Press, Princeton, NJ, 1979.

[12] F. Ricci and E. M. Stein, Harmonic analysis on nilpotent groups and singular integrals, I. Oscillatory integrals, J. Func. Anal. 73 (1987), 179-194.

[13] F. Ricci and E. M. Stein, Harmonic analysis on nilpotent groups and singular integrals, II. Singular kernels supported on submanifolds, J. Func. Anal. 78 (1988), $56-84$.

[14] N. Rivière, Singular integrals and multiplier operators, Ark. Mat. 9 (1971), 243-278.

[15] S. Sato, Estimates for singular integrals and extrapolation, Studia Math. 192 (2009), 219-233.

[16] S. Sato, Estimates for singular integrals along surfaces of revolution, J. Aust. Math. Soc. 86 (2009), 413-430.

[17] S. Sato, A note on $L^{p}$ estimates for singular integrals, Sci. Math. Jpn. 71 (2010), $343-348$.

[18] S. Sato, Estimates for singular integrals on homogeneous groups, J. Math. Anal. Appl. 400 (2013), 311-330.

[19] E. M. Stein, Harmonic Analysis: Real-Variable Methods, Orthogonality and Oscillatory Integrals, Princeton University Press, Princeton, NJ, 1993.

[20] E. M. Stein and S. Wainger, Problems in harmonic analysis related to curvature, Bull. Amer. Math. Soc. 84 (1978), 1239-1295.

[21] T. Tao, The weak-type $(1,1)$ of $L \log L$ homogeneous convolution operator, Indiana Univ. Math. J. 48 (1999), 1547-1584.

School of Mathematical Sciences, Laboratory of Mathematics and ComPlex Systems (BNU), Ministry of Education, Beijing Normal University, BeiJing, 100875 P. R. OF ChinA

E-mail address: dingy@bnu.edu.cn

Department of Mathematics, Faculty of Education, Kanazawa UniverSITY, KANAZAWA 920-1192, JAPAN

E-mail address: shuichi@kenroku. kanazawa-u.ac.jp 